\title{
Gap junctions promote synchronous activities in a network of inhibitory interneurons
}

\author{
A. Di Garbo*, A. Panarese, S. Chillemi \\ Istituto di Biofisica CNR, Sezione di Pisa,Via G. Moruzzi 1, Pisa 56124, Italy
}

\begin{abstract}
By using a single compartment biophysical model of a fast spiking interneuron the synchronization properties of a pair of cells, coupled by electrical and inhibitory synapses, are investigated. The inhibitory and excitatory synaptic couplings are modeled in order to reproduce the experimental time course of the corresponding currents. It is shown that increasing the conductance value of the electrical synapses enhances the synchronization between the spike trains of the two cells. Moreover, increasing either the decay time constant of the inhibitory current or the firing frequency of the cells favours the emergence of synchronous discharges.
\end{abstract}

(C) 2004 Elsevier Ireland Ltd. All rights reserved.

Keywords: Synchronization; Interneuron; Inhibitory; Excitatory; Gap junctions

\section{Introduction}

Recent experimental findings suggest that neocortical and hippocampal interneurons innervating the perisomatic region of pyramidal cells coordinate neural oscillatory activity (Fisahn and McBain, 2001; Galarreta and Hestrin, 2001a). Moreover, the synchronous firing of a neural population in the gamma frequency band $(30-100 \mathrm{~Hz})$ seems to be associated to cognitive functions (Gray et al., 1989; Csicsvari et al., 2003).

It was found that in the neocortex of young animals the fast spiking (FS) and the low-threshold spiking (LTS) inhibitory interneurons are interconnected by

\footnotetext{
* Corresponding author. Tel.: +39050 3152577; fax: +390503152760 .

E-mail address: digarbo@ib.cnr.it (A. Di Garbo).
}

electrical synapses too (Galarreta and Hestrin, 1999; Gibson et al., 1999; Galarreta and Hestrin, 2002). The relevance of the electrical synapses for the generation of the synchronous neural activity comes from the experimental finding that impairing the electrical synapses between cortical interneurons disrupts synchronous oscillations in the gamma frequency band (Deans et al., 2001; Hormuzdi et al., 2001). At present the specific functional role of the activity patterns of FS and LTS interneurons for cortical information processing are not well known. However, in a recent study it was found that FS cells are strongly excited by thalamocortical inputs, while the LTS cells are rarely and weakly affected by them (Beierlein et al., 2003). Thus, it was suggested that these two groups of interneurons are functionally distinct sources of neocortical inhibition. Moreover, additional experimental findings sug- 
gest that FS cells may play a relevant role in the detection of synchronous activity (Galarreta and Hestrin, 2001b).

From a the theoretical point of view, it was shown that a population of mutually inhibiting interneurons can generate synchronous oscillatory activity provided that specific constraints on the time course of the postsynaptic inhibitory conductance, tonic excitatory drive etc. are satisfied (Wang and Rinzel, 1992; Van Vreeswijk et al., 1995; Whittington et al., 1995; Wang and Buzsaki, 1996; White et al., 1998). At present the hot problem is to understand the functional role of the presence of electrical coupling in a inhibitory network (Chow and Kopell, 2000; Traub et al., 2001; Bartos et al., 2002; Di Garbo et al., 2002; Pfeuty et al., 2003; Lewis and Rinzel, 2003) and, in the Lewis and Rinzel (2003) paper, the existence and stability of the phase locking states of a pair of identical leaky integrate-andfire (LIF) models, coupled by electrical and chemical synapses, were investigated theoretically.

In our paper, we address a study similar to that done in (Lewis and Rinzel, 2003) by using a conductancebased model for each FS cell. One of the aims of our work is to study how electrical and inhibitory synapses affect the synchronization properties of a pair of coupled FS cells and compare our results with the LIF ones. Our contribution differs from that of the cited paper, and the main differences are the following: (a) we build a new conductance-based model of a single FS interneuron to meet at best its relevant biophysical properties (excitability, input resistance, duration and amplitude of the action potential, afterhyperpolarization, and resting potential); (b) we model synaptic inputs in order to fit the experimental time course of the inhibitory (excitatory) postsynaptic current between FS cells (respectively from pyramidal to FS cells). Lastly, for a pair of coupled LIF, the reduction to the phase model is carried out analytically, while for our conductance-based model of coupled FS cells, numerically alone.

In the weak coupling limit our results agree qualitatively with those of the cited paper, but some differences were found. For instance, we will show that, also increasing the intensity of the electrical conductance with slow synapses promotes synchronization. Unlike the Lewis and Rinzel (2003) paper, in our contribution the synchronization properties of a pair of coupled FS cells were investigated in the presence of synaptic inputs with poisson distribution. In this case and without the weak limit approximation, we will show that the synchronization level of a pair of coupled FS cells, each one receiving independent excitatory synaptic bombardment from pyramidal neurons, is enhanced as the electrical conductance increases (both for fast and slow synapses).

\section{Materials and methods}

\subsection{Experimental data on FS interneurons}

FS interneurons are not capable of generating repetitive firing of arbitrary low frequency when they are injected with a constant current (Erisir et al., 1999). Thus, they have the type II excitability property (Rinzel and Ermentrout, 1989).

The following experimental data on FS cells were obtained in vitro from the somatosensory cortex of adult mice and are expressed as mean \pm standard error of the mean (S.E.M.): FS interneurons have high firing rates (up to $\sim 210 \mathrm{~Hz}$ ), average resting membrane potential of $(-72 \pm 1) \mathrm{mV}$ and input resistance of $(89.2 \pm 2.9) \mathrm{M} \Omega$; their action potential has a mean half-width of $(0.35 \pm 0.01) \mathrm{ms}$, average amplitude of $(61 \pm 2) \mathrm{mV}$ and afterhyperpolarization of $(25.4 \pm 0.6) \mathrm{mV}$ (Galarreta and Hestrin, 2002). The mean value of the electrical conductance between two FS cells is $\left\langle G_{\mathrm{El}}\right\rangle \pm \Delta G_{\mathrm{El}}=(0.203 \pm 0.044) \mathrm{nS}$, the maximal conductance of unitary GABAergic synapses is $\left\langle G_{\mathrm{GABA}}\right\rangle \pm \Delta G_{\mathrm{GABA}}=(0.8 \pm 0.26) \mathrm{nS}$ with a reversal potential of $-80 \mathrm{mV}$, the mean value of the decay time constant of the inhibitory postsynaptic current (IPSC) is $\left\langle\tau_{\operatorname{Exp}}\right\rangle \pm \Delta \tau=(2.6 \pm 0.2) \mathrm{ms}$ (Galarreta and Hestrin, 2002). We used the experimental time course of the average (50 trials) IPSC to determine, by standard fitting method, the simplest mathematical form capable to capture the rising and decay kinetics of the IPSC. The adopted function was $A\left[1-\exp \left(-t / \tau_{\text {Rise }}\right)\right]$ $\exp \left(-t / \tau_{\text {Decay }}\right)$ with $A=95.451 p \mathrm{~A}, \tau_{\text {Rise }}=0.289 \mathrm{~ms}$, $\tau_{\text {Decay }}=2.115 \mathrm{~ms}$.

\subsection{Biophysical model of FS cells}

Models of Hodgkin-Huxley type of a FS cell were proposed by several authors (Wang and Buzsaki, 1996; Erisir et al., 1999; Durstewitz et al., 2000). Here we will describe the activity of each FS cell with a single 
compartment biophysical model derived from a model proposed recently (Durstewitz et al., 2000): it consists of an isopotential sphere (the soma) of radius $R=7.5 \mu \mathrm{m}$. To better match the main experimental electrophysiological properties of an FS cell the conductances and kinetics of the ionic currents of that model were modified, and the new model reads:

$$
\begin{aligned}
& \frac{C \mathrm{~d} V}{\mathrm{~d} t}=I_{\mathrm{E}}-g_{\mathrm{Na}} m^{3} h\left(V-V_{\mathrm{Na}}\right)-g_{\mathrm{K}} n^{4}\left(V-V_{\mathrm{K}}\right) \\
& \quad-g_{\mathrm{L}}\left(V-V_{\mathrm{L}}\right) \\
& \frac{\mathrm{d} x}{\mathrm{~d} t}=\frac{\left(x_{\infty}-x\right)}{t_{x}}, \quad x_{\infty}=\frac{\alpha_{x}}{\left(\alpha_{x}+\beta_{x}\right)}, \\
& \tau_{x}=\frac{1}{\left(\alpha_{x}+\alpha \beta_{x}\right)}, \quad x=m, h, n
\end{aligned}
$$

where $\mathrm{C}=1 \mu \mathrm{F} / \mathrm{cm}^{2}, I_{\mathrm{E}}$ is the external stimulation current, and $\alpha_{m}=4.2 \exp [(V+34.5) / 11.57], \beta_{m}=4.2$ $\exp [-(V+34.5) / 27], \quad \alpha_{h}=0.09 \quad \exp [-(V+45) / 33]$, $\beta_{h}=0.09 \exp [(V+45) / 12.2], \alpha_{n}=0.3 \exp [(V+35) /$ 13.83], $\beta_{n}=0.3 \exp [-(V+35) / 14.06]$. The maximal specific conductances and the reversal potentials are, respectively: $g_{\mathrm{Na}}=52 \mathrm{mS} / \mathrm{cm}^{2}, \quad g_{\mathrm{K}}=250 \mathrm{mS} / \mathrm{cm}^{2}$, $g_{\mathrm{L}}=1.6 \mathrm{mS} / \mathrm{cm}^{2}$ and $V_{\mathrm{Na}}=58 \mathrm{mV}, \quad V_{\mathrm{K}}=-90 \mathrm{mV}$ and $V_{\mathrm{L}}=-72 \mathrm{mV}$. In this model, the onset of periodic firing occurs through a Hopf bifurcation for $I_{\mathrm{E}} \cong 28.7 \mu \mathrm{A} / \mathrm{cm}^{2}$ with a well defined frequency $(\sim 37 \mathrm{~Hz})$, according to the type II excitability property (Rinzel and Ermentrout, 1989). The other properties of the model are the following: resting membrane potential at $-72 \mathrm{mV}$; input resistance $\sim 88 \mathrm{M} \Omega$; action potential amplitude $\sim 65 \mathrm{mV}$; afterhyperpolarization amplitude $\sim 27 \mathrm{mV}$ and action potential half-width $\sim 0.1 \mathrm{~ms}$.

\subsection{Modelling the synaptic coupling}

The fitted experimental time course of the IPSC, $I_{\mathrm{GABA}}(t)=A\left[1-\exp \left(-t / \tau_{\text {Rise }}\right)\right] \exp \left(-t / \tau_{\text {Decay }}\right)$, is used here to model the inhibitory synapse between FS cells: $I_{\text {Sy }}=g_{\text {Sy }} s(t)\left(V_{\text {Post }}-V_{\text {Rev }}\right)$, where $g_{\text {Sy }}$ is the specific maximal conductance of the inhibitory synapse (in $\mathrm{mS} / \mathrm{cm}^{2}$ unit), $s(t)=I_{\mathrm{GABA}}(t) / \mathrm{Max}\left(I_{\mathrm{GABA}}\right.$ $(t)), \quad \operatorname{Max}\left(I_{\mathrm{GABA}} \quad(t)\right)=A\left[\tau_{\text {Decay }} /\left(\tau_{\text {Rise }}+\tau_{\text {Decay }}\right)\right]$ $\left[\tau_{\text {Rise }} /\left(\tau_{\text {Rise }}+\tau_{\text {Decay }}\right)\right] \tau_{\text {Rise }} / \tau_{\text {Decay }}$ and $V_{\text {Rev }}=$ $-80 \mathrm{mV}$. In the following we will keep $\tau_{\text {Rise }}=0.289 \mathrm{~ms}$ and $\tau_{\text {Decay }}=\left\langle\tau_{\text {Exp }}\right\rangle=2.6 \mathrm{~ms}$; moreover, where stated, the $\tau_{\text {Decay }}$ value will be varied within a given interval.

To get a physiological estimate of $g_{\text {Sy }}$ we proceed as follows: from the experimental value of $\left\langle G_{\mathrm{GABA}}\right\rangle$ we get $g_{\mathrm{Sy}}=\left\langle G_{\mathrm{GABA}}\right\rangle / 4 \pi R^{2}=0.1122 \mathrm{mS} / \mathrm{cm}^{2}$. Besides, if a presynaptic interneuron generated $N$ spikes at times $t_{j}(j=1,2, \ldots N)$ the magnitude of the inhibitory current at time $t>t_{N}$ in the postsynaptic cell it is $I_{\mathrm{Sy}}=g_{\mathrm{Sy}} \sum_{j=1}^{N} s\left(t-t_{j}\right)\left(V_{\text {Post }}(t)-V_{\text {Rev }}\right)$. Here, each value of $t_{j}$ represents the time when membrane potential, $V(t)$, crosses a well defined threshold with positive derivative.

The electrical synapse is modelled as $I_{\mathrm{El}}=g_{\mathrm{El}}$ $\left(V_{\text {Post }}-V_{\text {Pre }}\right)$ where $g_{\mathrm{El}}$ is the maximal specific conductance. To get values of $g_{\mathrm{El}}$ within the physiological range we proceed as for inhibitory synapse: from the experimental value of $\left\langle G_{\mathrm{El}}\right\rangle$ it follows that $g_{\mathrm{El}}=\left\langle G_{\mathrm{El}}\right\rangle / 4 \pi R^{2}=0.0286 \mathrm{mS} / \mathrm{cm}^{2}$. Lastly, the two synaptic current $I_{\mathrm{Sy}}, I_{\mathrm{El}}$ defined so far, affect the dynamics of a FS interneuron model by adding the term $\left(-I_{\mathrm{Sy}}-I_{\mathrm{El}}\right)$ to the right hand side of Eq. (1a). In this paper, $g_{S y}=0.11 \mathrm{mS} / \mathrm{cm}^{2}$ it is the adopted value of the maximal conductance of the inhibitory synapse, while the values of $g_{\mathrm{El}}$ will be varied in the range from 0 to $0.1 \mathrm{mS} / \mathrm{cm}^{2}$.

Some typical discharge patterns of a pair of FS cells connected by inhibitory synapses are reported in Fig. 1. They were obtained by numerically solving Eq. (1) with $g_{\mathrm{Sy}}=0.11 \mathrm{mS} / \mathrm{cm}^{2}$ and $g_{\mathrm{El}}=0 \mathrm{mS} / \mathrm{cm}^{2}$. It can be seen (top and middle panels) that for low $I_{\mathrm{E}}$ values the system exhibits bistability, while for higher values only synchronous states are stable. Similar results were found either by adding electrical synapses and for mixed coupling.

\subsection{Weak coupling limit}

Let us consider a pair of coupled non-linear oscillators:

$$
\begin{array}{r}
\frac{\mathrm{d} X_{i}}{\mathrm{~d} t}=F\left(X_{i}\right)+\varepsilon G_{i}\left(X_{i}, X_{j}\right), \quad X_{i} \in R^{n}, \\
i=1,2, \quad i \neq j
\end{array}
$$

where $\mathrm{X}_{i}(t)$ defines the state of the $i$-th oscillator and $\varepsilon G_{i}\left(X_{i}, X_{j}\right)(\varepsilon \ll 1)$ is the coupling term. For the FS cell model, defined in Eq. 1 , it is $X_{i}=(V$, $m, h, n)$ and $\varepsilon G_{i}\left(X_{i}, X_{j}\right)=\left(-I_{\mathrm{Sy}}(i)-I_{\mathrm{El}}(i), 0,0\right.$, 

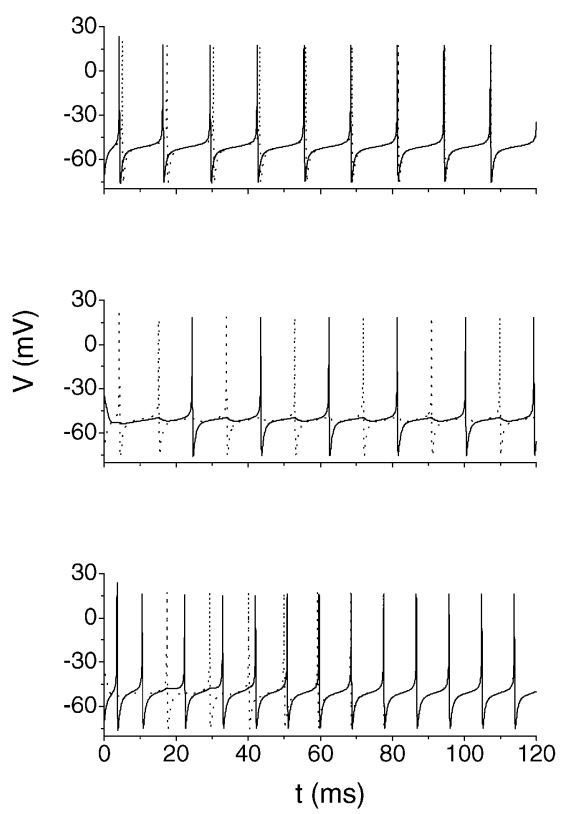

Fig. 1. Typical discharge patters of a pair of FS cell model coupled by reciprocal inhibitory synapses alone. Top and middle panels: $I_{\mathrm{E}}=29.6 \mu \mathrm{A} / \mathrm{cm}^{2}$, but different initial conditions; bottom panel: $I_{\mathrm{E}}=30.6 \mu \mathrm{A} / \mathrm{cm}^{2}$. For all panels it is $\tau_{\text {Decay }}=\left\langle\tau_{\text {Exp }}\right\rangle=2.6 \mathrm{~ms}$.

0 ). Then, assuming that for $\varepsilon=0$, each oscillator possesses a stable limit cycle $X_{\mathrm{o}}(t)$ of period $T$, in the weak coupling approximation the state of each oscillator is defined by its phase $\theta_{i}(i=1,2)$ and the corresponding dynamical behaviour is determined by the following equations: $\mathrm{d} \theta_{1} / \mathrm{d} t=1+\varepsilon H_{1}(\phi)$ and $\mathrm{d} \theta_{2} / \mathrm{d} t=1+\varepsilon H_{2}(-\phi), \quad$ where $\quad \phi=\theta_{2}-$ $\theta_{1}, \quad H_{1}(\phi)=\frac{1}{T} \int_{0}^{T} Y(t) \cdot G_{1}\left(X_{\mathrm{o}}(t), \quad X_{\mathrm{o}}(t+\phi)\right) \mathrm{d} t$, $H_{2}(-\phi)=\frac{1}{T} \int_{0}^{T} Y(t) \cdot G_{2}\left(X_{\mathrm{o}}(t), \quad X_{\mathrm{o}}(t-\phi)\right) \mathrm{d} t$ and they are $T$-periodic functions (Ermentrout, 1998). The vector $Y(t)$ is the normalized $T$-periodic solution of the adjoint equation $\mathrm{d} Y(t) / \mathrm{d} t=-\left[J\left(\mathrm{X}_{\mathrm{o}}(t)\right)\right]^{T} Y(t)$ with $\int_{0}^{T} Y(t) \cdot X_{\mathrm{o}}(t) \mathrm{d} t=1$, and $J^{T}$ is the transpose of the Jacobian matrix $J=\partial F_{i}\left(X_{\mathrm{o}}\right) / \partial X_{j}$.

For a pair of identical FS cells coupled by inhibitory synapses it is $H_{1, S}(\phi)=\frac{1}{T} \int_{0}^{T} Y_{1}(t) s_{\mathrm{O}}(t+\phi)\left(V_{\mathrm{Rev}}-\right.$ $\left.V_{\mathrm{o}}(t)\right) \mathrm{d} t$ and $H_{2, S}(-\phi)=\frac{1}{T} \int_{0}^{T} Y_{1}(t) s_{\mathrm{o}}(t-\phi)\left(V_{\mathrm{Rev}}-\right.$ $\left.V_{\mathrm{o}}(t)\right) \mathrm{d} t$, where $Y_{1}(t)$ is the first component of $\mathrm{Y}(t)$, $V_{\mathrm{o}}(t)$ is the membrane voltage of the unperturbed FS cell model and $s_{0}(t)$ is the $T$-periodic function when the membrane voltage of the presynaptic cell is given by $V_{\mathrm{o}}(t)$. The time evolution of $\phi$ is deter- mined by $\mathrm{d} \phi / \mathrm{d} t=\varepsilon\left[H_{2, S}(-\phi)-H_{1, S}(\phi)\right]=-\varepsilon D_{S}(\phi)$ $\left(\varepsilon=g_{\mathrm{Sy}}\right)$ and the phase locked states can be determined by searching the solutions of the equation $D_{S}\left(\phi^{*}\right)=0$. A phase locked state is characterized by a constant phase difference, $\phi^{*}$, between the two oscillators and it will be stable (or unstable) according as $\mathrm{d} D_{S} / \mathrm{d} \phi>0\left(\mathrm{~d} D_{S} / \mathrm{d} \phi<0\right)$. Similarly for electrical synapses it is $H_{1, \mathrm{E}}(\phi)=\frac{1}{T} \int_{0}^{T} Y_{1}(t)\left(V_{\mathrm{o}}(t+\phi)-\right.$ $\left.V_{\mathrm{o}}(t)\right) \mathrm{d} t, H_{2, \mathrm{E}}(-\phi)=\frac{1}{T} \int_{0}^{T} Y_{1}(t)\left(V_{\mathrm{o}}(t-\phi)-V_{\mathrm{o}}(t)\right) \mathrm{d} t$ and $\mathrm{d} \phi / \mathrm{d} t=-\varepsilon D_{\mathrm{E}}(\phi) \quad\left(\varepsilon=g_{\mathrm{El}}\right)$. Lastly, for mixed coupling the time evolution of $\phi$ is determined by $\mathrm{d} \phi / \mathrm{d} t=-\varepsilon\left[D_{S}(\phi)+\frac{g_{\mathrm{El}}}{g_{\mathrm{Sy}}} D_{\mathrm{E}}(\phi)\right]=-\varepsilon D(\phi)\left(\varepsilon=g_{\mathrm{Sy}}\right)$ and the phase locked states are defined as the solutions of the equation $D\left(\phi^{*}\right)=0$.

\subsection{Measures of the synchronization between spike trains}

In numerical simulations of a pair of FS cells subject to Poissonian synaptic bombardment we used the normalized cross-correlation histogram to quantify their synchronization level: this measure is denoted by $\Gamma(t)$ and, the adopted time bin, is $1 \mathrm{~ms}$.

\section{Results}

\subsection{Weak coupling limit}

Firstly let us consider a pair of identical FS cells reciprocally coupled by inhibitory synapses alone to investigate the existence and stability of phase locked states as the decay time constant $\tau_{\text {Decay }}$ of IPSC is varied. The stimulation current $I_{\mathrm{E}}$ is the same for both interneurons and the results are reported in Fig. 2: there is a critical value $\tau_{\mathrm{C}}$ of the decay time constant such that for $\tau_{\text {Decay }}<\tau_{\mathrm{C}}$ there is bistability (synchrony and anti-synchrony), while for $\tau_{\text {Decay }}>\tau_{\mathrm{C}}$ the only stable states are the synchronous ones. From a dynamical point of view for $\tau_{\text {Decay }}=\tau_{\mathrm{C}}$, a sub-critical pitchfork bifurcation occurs: the anti-phase state loses the stability and two unstable branches, behaving as separatrices between anti-phase and in-phase states, appear. Thus, for a fixed $\tau_{\text {Decay }}$, the quantity $2-2 \phi(\tau)$ gives a rough estimation of the probability to reach asymptotically a synchronous regime for random initial conditions $(\phi(\tau)$ being the phase of the corresponding state in the top 

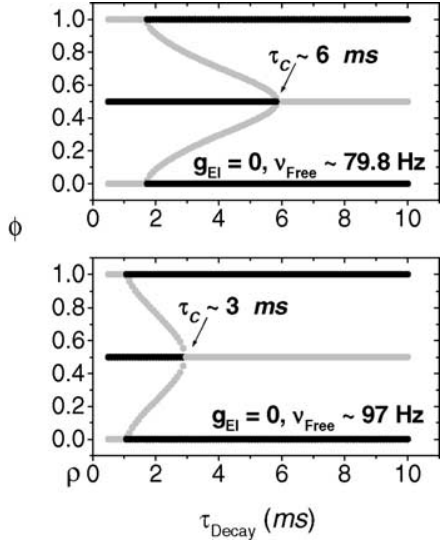

Fig. 2. Bifurcation diagram of the phase difference against $\tau_{\text {Decay }}$ for a pair of FS cells weakly coupled by inhibitory synapses alone. Top panel: $I_{\mathrm{E}}=29.6 \mu \mathrm{A} / \mathrm{cm}^{2}$; bottom panel: $I_{\mathrm{E}}=30 \mu \mathrm{A} / \mathrm{cm}^{2}$. For both panels $\nu_{\text {Free }}$ is the free firing frequency of each cell for the used $I_{\mathrm{E}}$ values.

unstable branch). As the value of the stimulation current $I_{\mathrm{E}}$ increases synchronization is promoted (bottom panel of Fig. 2) and $\tau_{\mathrm{C}}$ gets physiologically relevant values $\left(\tau_{\mathrm{C}} \leq 3 \mathrm{~ms}\right)$.

The existence and stability of phase locked states in a pair of FS cell models were investigated against $I_{\mathrm{E}}$ by keeping $\tau_{\text {Decay }}=\left\langle\tau_{\text {Exp }}\right\rangle=2.6 \mathrm{~ms}$. In the top panel of Fig. 3 the stationary values of $\phi$ are reported for the case $g_{\mathrm{El}} \neq 0, g_{\mathrm{Sy}}=0$. There is a critical value of the stimulation current, $I_{\mathrm{C}}$, such that for $I_{\mathrm{E}}<I_{\mathrm{C}}$ bistability occurs, while for $I_{\mathrm{E}}>I_{\mathrm{C}}$ only the synchronous state is stable. The stationary values of $\phi$ for the case $g_{\mathrm{El}}=0, g_{\mathrm{Sy}} \neq 0$ are reported in the middle panel of Fig. 3 and, also in this case, there is a critical value of the stimulation current that separates the two regimes, bistability and synchrony. The results obtained when the two FS cells are coupled by both inhibitory and electrical synapses are shown in the bottom panel of Fig. 3. In this case, the bifurcation diagram is intermediate between those shown in the top and middle panels.

To quantify the effects of electrical coupling on the synchronization level of a pair of FS cell models, we used the following protocol: for any fixed value of the ratio $Q=g_{\mathrm{E}} / g_{\mathrm{Sy}}$ we evaluated $I_{\mathrm{C}}(Q)$ from the associated bifurcation diagram (for $I_{\mathrm{E}}$ ranging in the interval from 29.2 to $30.5 \mu \mathrm{A} / \mathrm{cm}^{2}$ ); next, we considered the rectangle with unity height and base $I_{\mathrm{C}}(Q)-29.2$ and calculated the area $A_{\mathrm{UB}}$, of the region contained within the unstable branches. Then, the prob-
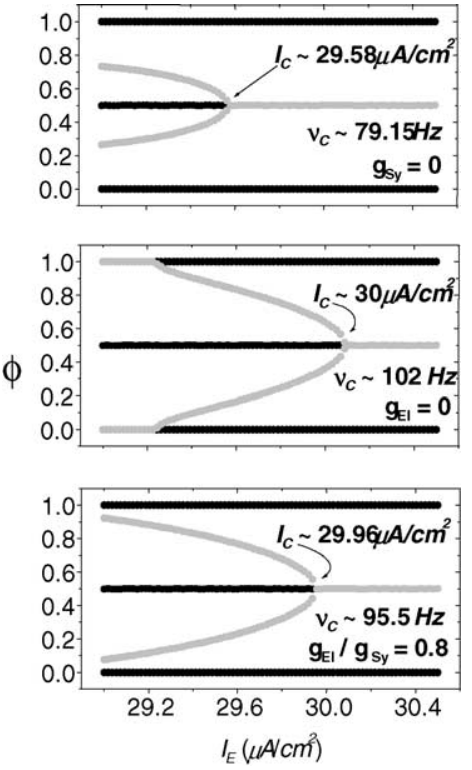

Fig. 3. Bifurcation diagram of the phase difference against $I_{\mathrm{E}}$ for a pair of coupled FS cells. Top panel: $g_{\mathrm{Sy}}=0 \mathrm{mS} / \mathrm{cm}^{2}$; middle panel: $g_{\mathrm{El}}=0 \mathrm{mS} / \mathrm{cm}^{2}$; bottom panel: $g_{\mathrm{El}} / g_{\mathrm{Sy}}=0.8$. For all panels it is $\tau_{\text {Decay }}=\left\langle\tau_{\text {Exp }}>=2.6 \mathrm{~ms}\right.$.

ability of synchronous firing was estimated as follows: $P_{S}=1-A_{\mathrm{UB}} / A_{\mathrm{Tot}}$, being $A_{\mathrm{Tot}}=I_{\mathrm{C}}(Q)-29.2$. The $P_{S}$ values are plotted in Fig. 4 against $g_{\mathrm{El}} / g_{\mathrm{Sy}}$ for the cases $\tau_{\text {Decay }}=5.2 \mathrm{~ms}$ and $\tau_{\text {Decay }}=2.6 \mathrm{~ms}$. The corresponding curves exhibit an increasing trend and this means that increasing the value of $g_{\mathrm{El}}$ promotes synchronization. The high values of $P_{S}$ for $\tau_{\text {Decay }}=5.2 \mathrm{~ms}$ can be explained by the fact that slow synaptic current enhances synchronization (see Fig. 2). Our results are qualitatively in keeping for fast synapses, but at odds for slow ones, with those obtained by Lewis and Rinzel (2003).

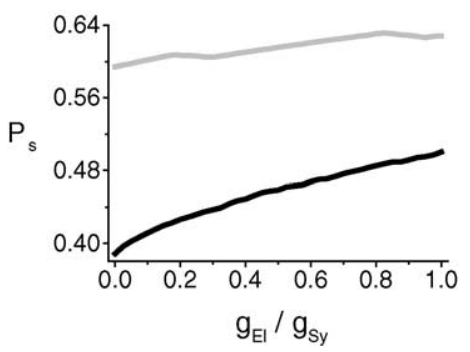

Fig. 4. Probability of synchronous firing of a pair of FS cell models coupled by electrical and chemical synapses against $g_{\mathrm{El}} / g_{\mathrm{Sy}}$. Black line: $\tau_{\text {Decay }}=2.6 \mathrm{~ms}$; grey line: $\tau_{\text {Decay }}=5.2 \mathrm{~ms}$. 
The above findings were obtained for symmetrical inhibitory coupling. We found that using two different values for the conductances of the inhibitory synapses destroys, as it is expected, the symmetry of the bifurcation diagrams shown in the previous Figures (data not shown). However, when the intensity of the electrical coupling is increased, the synchronization is enhanced: i.e., the probability to get synchronous states starting with arbitrary initial conditions increases and the symmetry of the bifurcation diagram is partially recovered (data not shown).

\subsection{Full network simulations}

FS interneurons receive excitatory inputs from pyramidal neurons and recently the properties of these synapses were studied experimentally (Galarreta and Hestrin, 2001b). Thus, we used these experimental data on the excitatory postsynaptic currents (EPSC) from pyramidal to FS cells to build a more realistic model of a pair of coupled FS interneurons. To model the EPSC we followed the same approach described in section 2.3 for the IPSC. Poissonian spike trains (of $1000 \mathrm{~Hz}$ rate) were generated, convolved with the experimental waveform of EPSC and added to the right hand side of Eq. (1a). Then the corresponding equations were integrated numerically.

For the discharges recorded from neocortical FS cell in in vitro experiments, a very low value of the coefficient of variation $(\mathrm{CV})$ of the interspike intervals (ISIs) has been measured: $\mathrm{CV}=0.086$ (Galarreta and Hestrin, 2002). Thus, the amplitude of the EPSC for our computer experiments was fixed in order to yield for the uncoupled FS cell a CV value of the same magnitude as the experimental one. By adopting $g_{\text {EPSC }}=0.0047 \mathrm{mS} / \mathrm{cm}^{2}$ for the maximal conductance of the excitatory synapses, we got $\mathrm{CV} \sim 0.085$. The plots of $\Gamma(t)$ are shown in Fig. 5: for $g_{\mathrm{El}}=g_{\mathrm{Sy}}=0$ the corresponding $\Gamma$ curve is flat and the discharges of the two interneurons are uncorrelated as expected (thin black line). The introduction of the inhibitory coupling leads to the emergence of an oscillatory activity (thick black line). The addition of electrical coupling reinforces the oscillatory activity and promotes the synchronization by increasing the number of events in which action potentials are generated within $1 \mathrm{~ms}$ time scale (grey line).

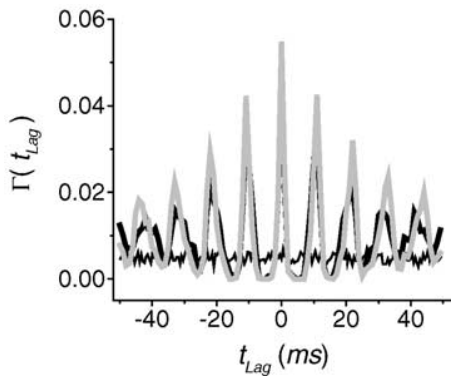

Fig. 5. Normalized cross-correlation histograms for a pair of coupled FS cell models when each cell receives uncorrelated Poisson distributed excitatory inputs of $1000 \mathrm{~Hz}$ rate and maximal amplitude $g_{\text {EPSC }}=0.0047 \mathrm{mS} / \mathrm{cm}^{2}$. Thin black line: $g_{\mathrm{El}}=g_{\mathrm{Sy}}=0$; thick black line: $g_{\mathrm{El}}=0, g_{\mathrm{Sy}}=0.11 \mathrm{mS} / \mathrm{cm}^{2}$; grey line: $g_{\mathrm{E}}=0.05 \mathrm{mS} / \mathrm{cm}^{2}$, $g_{\mathrm{Sy}}=0.11 \mathrm{mS} / \mathrm{cm}^{2}$. For both cells it is $I_{\mathrm{E}}=29.4 \mu \mathrm{A} / \mathrm{cm}^{2}$ and $\tau_{\text {Decay }}=\left\langle\tau_{\text {Exp }}\right\rangle=2.6 \mathrm{~ms}$.

A systematic investigation of the effects of the electrical coupling on the synchronization properties of a pair of coupled FS cells subject to uncorrelated EPCSs was performed. In particular we were interested to follow the changes of the average firing frequency $\langle\nu\rangle$, $\mathrm{CV}$ and $\Gamma(0)$ as $g_{\mathrm{El}}$ is increased and the corresponding results are reported in Fig. 6.
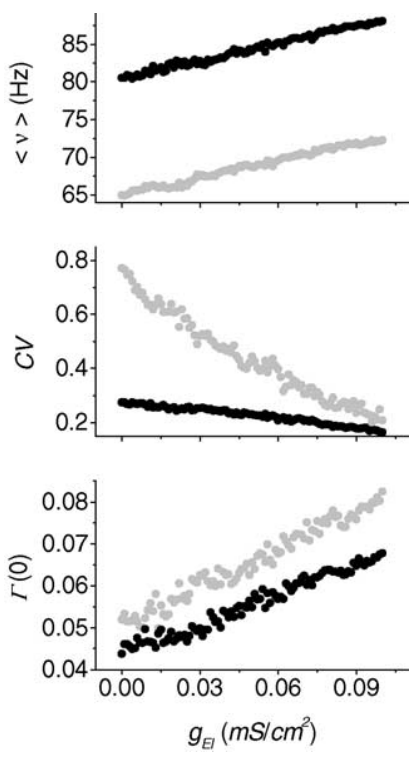

Fig. 6. Dependence of $\langle\nu\rangle, \mathrm{CV}$ and $\Gamma(0)$ on the electrical coupling conductance. Black solid circles: $\tau_{\text {Decay }}=2.6 \mathrm{~ms}$; grey solid circles: $\tau_{\text {Decay }}=5.2 \mathrm{~ms}$. For all panels it is $g_{\text {Sy }}=0.11 \mathrm{mS} / \mathrm{cm}^{2}$, $g_{\mathrm{EPSC}}=0.0047 \mathrm{mS} / \mathrm{cm}^{2}$ and $I_{\mathrm{E}}=29.4 \mu \mathrm{A} / \mathrm{cm}^{2}$ for both cells. 
For $\tau_{\text {Decay }}=\left\langle\tau_{\text {Exp }}\right\rangle=2.6 \mathrm{~ms}$ the variable $\langle\nu\rangle$ is proportional to $g_{\mathrm{El}}$ and a linear fitting protocol gives a correlation coefficient $r=0.99(p<0.0001)$. The monotonic increase of $\langle\nu\rangle$ can be explained by the fact that the presence of the electrical coupling increases the probability that a presynaptic spike evokes an additional spike in the postsynaptic cell. The middle panel of Fig. 6 shows that $C V$ is inversely proportional to $g_{\mathrm{El}}$ $(r=-0.98, p<0.0001)$. This means that increasing $g_{\mathrm{El}}$ enhances the regularity of the firing activity of each cell. The plot of the $\Gamma(0)$ values against $g_{\mathrm{El}}$ in the bottom panel of Fig. 6 implies that the synchronization between the spike trains of the two FS interneurons is promoted by increasing $g_{\mathrm{El}}(r=0.98, p<0.0001)$. An interesting feature exhibited by the plot of $\Gamma(0)$ values is the presence of a threshold: for $g_{\mathrm{El}} \leq 0.025 \mathrm{mS} / \mathrm{cm}^{2}$ the average synchronization level is small, while it is higher for $g_{\mathrm{El}}>0.025 \mathrm{mS} / \mathrm{cm}^{2}$. To assess quantitatively this point we performed linear fits to the plot in the two intervals of $g_{\mathrm{El}}$ values: $\left(0: 0.025 \mathrm{mS} / \mathrm{cm}^{2}\right)$ and $(0.026$ : $0.051 \mathrm{mS} / \mathrm{cm}^{2}$ ), each containing the same number of data points (\# 26). For the first interval the correlation coefficient was $r=0.55(p=0.003)$, while for the second one it was $r=0.89(p<0.0001)$.

Similar results were found for the case $\tau_{\text {Decay }}=5.2 \mathrm{~ms}$ (see Fig. 6, grey solid circles); for the explored range of $g_{\mathrm{El}}$ values the average discharge frequency of the cells is always lower than in the former case, but its increasing trend against $g_{\mathrm{El}}$ is maintained. The $\mathrm{CV}$ plot shows that increasing the decay time constant of the inhibitory current leads to a deterioration of the regularity of the cell discharges. The $\Gamma(0)$ shows that the probability to get a synchronous discharge is higher than in the former case as predicted from the weak coupling limit results (see Fig. 4).

We conclude with two remarks: firstly, that the interval of $g_{\mathrm{El}}$ values where the average synchronization level is high is physiologically relevant; secondly, that a similar threshold phenomenon was recently found for a network of multicompartment pyramidal cells and interneurons (Traub et al., 2001) with a $g_{\mathrm{El}}$ threshold value of $\sim 0.3 \mathrm{nS}$ (that, for our model, corresponds to $g_{\mathrm{El}} \sim 0.044 \mathrm{mS} / \mathrm{cm}^{2}$ ).

The effects of the heterogeneity, $I_{\mathrm{E}}(1) \neq I_{\mathrm{E}}(2)$, on the network coherence were investigated too. It was found that there is a decrease of the coherence level between the cells, but increasing the intensity of the electrical coupling prevents these desynchronizing effects (data not shown). Similar results were found by using two different values of the inhibitory conductances (data not shown).

\section{Conclusions}

We studied the synchronization phenomena occurring in a pair of FS cell models coupled by electrical and inhibitory synapses. Each inhibitory synapse was modelled by using the experimental time course of the IPSC. The work was carried out either analytically in the weak coupling limit or by direct numerical integration of the equations defining the network.

The main results obtained in the weak coupling limit are the following. In the case $g_{\mathrm{El}} \neq 0$ and $g_{\mathrm{Sy}}=0$ there is a critical value, $I_{\mathrm{C}}$, of the stimulation current $I_{\mathrm{E}}$ such that for $I_{\mathrm{E}}>I_{\mathrm{C}}$ only synchronous firing is stable, while for $I_{\mathrm{E}}<I_{\mathrm{C}}$ bistability regimes occur. A similar behaviour was found for the two cases $g_{\mathrm{El}}=0, g_{\mathrm{Sy}} \neq 0$ and $g_{\mathrm{El}} \neq 0, g_{\mathrm{Sy}} \neq 0$. For a given $I_{\mathrm{E}}$ value and $g_{\mathrm{El}}=0$, $g_{\text {Sy }} \neq 0$ there is a critical value $\tau_{\mathrm{C}}$ of $\tau_{\text {Decay }}$ such that for $\tau_{\text {Decay }}>\tau_{\mathrm{C}}$ only synchronous firing regimes occur, while for $\tau_{\text {Decay }}<\tau_{\mathrm{C}}$ bistability is dominant. In this case, for $I_{\mathrm{E}}>30 \mu \mathrm{A} / \mathrm{cm}^{2}$ the corresponding $\tau_{\mathrm{C}}$ values are physiologically relevant $\left(\tau_{\mathrm{C}}<3 \mathrm{~ms}\right)$. These findings imply that increasing either the stimulation current or the decay time constant of IPCS enhances the probability to get synchronous and stable firing regimes starting with arbitrary random initial conditions. These results are qualitatively in agreement with those obtained for a pair of coupled LIF models (Lewis and Rinzel, 2003).

For a pair of coupled LIF, it was found, in the weak coupling limit, that fast (slow) inhibitory synapses enhance (reduce) the probability of synchronous firing as the conductance of electrical synapses increases (Lewis and Rinzel, 2003). We performed a similar numerical experiment and found that for fast synapses $\left(\tau_{\text {Decay }}=2.6 \mathrm{~ms}\right)$ our results agree qualitatively with those obtained for a LIF model pair, while for slow $\left(\tau_{\text {Decay }}=5.2 \mathrm{~ms}\right)$ synapses they are conflicting. In fact for $\tau_{\text {Decay }}=5.2 \mathrm{~ms}$ we found that increasing $g_{\text {El }}$ promotes synchrony too, although the average slope of the plot of $P_{S}$ values (see Fig. 4) is lower than that corresponding to $\tau_{\text {Decay }}=2.6 \mathrm{~ms}$.

The study of the synchronization phenomena occurring in more realistic conditions was addressed by di- 
rect numerical integration of the equations modelling a pair of coupled FS interneurons. Each cell received uncorrelated Poisson distributed excitatory inputs (modelled by using the experimental time course of the EPSC from a pyramidal cell to an FS one) of $1000 \mathrm{~Hz}$ rate; the amplitude of these currents was chosen in order reproduce the CV of ISIs of an FS cell in in vitro conditions. By keeping $g_{S y}=0.11 \mathrm{mS} / \mathrm{cm}^{2}$ (as estimated from the experimental data) and $g_{\mathrm{El}}=0$ we found that the system of coupled cells generates coherent high frequency discharges. As the electrical coupling was set on we saw an enhancement of the synchronization level (see Fig. 5). The effects of the electrical coupling on the network dynamics were systematically investigated by plotting the values of the $\langle\nu\rangle$, of the $\mathrm{CV}$ and of the $\Gamma(0)$ against $g_{\mathrm{El}}$. We found, both for fast $\left(\tau_{\text {Decay }}=2.6 \mathrm{~ms}\right)$ and slow $\left(\tau_{\text {Decay }}=5.2 \mathrm{~ms}\right)$ synapses, that $\Gamma(0)$ and $\langle\nu\rangle(\mathrm{CV})$ exhibit an increasing (decreasing) trend as $g_{\mathrm{El}}$ increases. This means, in agreements with the results in the weak coupling limit, that the presence of the gap junctions in a pair of mutually inhibiting FS cell model enhance their synchronous firing.

Lastly, we showed that the presence of heterogeneity deteriorates the coherence levels of the network, however this effect can be balanced by increasing the $g_{\text {El }}$ value.

\section{Acknowledgements}

We thank Prof. S. Hestrin and Dr. M. Galarreta for providing us the experimental data on IPSC and EPSC acting on FS cell and for their helpful comments during the preparation of the manuscript.

\section{References}

Bartos, M., Vida, I., Frotscher, M., Meyer, A., Monyer, H., Geiger, J.R.P., Jonas, P., 2002. Fast synaptic inhibition promotes synchronized gamma oscillations in hippocampal interneuron networks. Proc. Natl. Acad. Sci. U.S.A. 99, 13222-13227.

Beierlein, M., Gibson, J.R., Connors, B.W., 2003. Two dynamically distinct inhibitory networks in layer 4 of the neocortex. J. Neurophysiol. 90, 2987-3000.

Chow, C.C., Kopell, N., 2000. Dynamics of spiking neurons with electrical coupling. Neural Comput. 12, 1643-1678.

Csicsvari, J., Jamieson, B., Wise, K.D., Buzsaki, G., 2003. Mechanisms of gamma oscillations in the hippocampus of the behaving rat. Neuron 37, 311-322.
Deans, M.R., Gibson, J.R., Sellitto, C., Connors, B.W., Paul, D.L., 2001. Synchronous activity of inhibitory networks in neocortex requires electrical synapses containing connexin36. Neuron 31, 477-485.

Di Garbo, A., Barbi, M., Chillemi, S., 2002. Synchronization in a network of fast-spiking interneurons. BioSystems 67, 45-53.

Durstewitz, D., Seamans, J.K., Sejnowski, T.J., 2000. Dopaminemediated stabilization of delay-period activity in a network model of prefrontal cortex. J. Neurophysiol. 83, 1733-1750.

Erisir, A., Lau, D., Rudy, B., Leonard, C.S., 1999. Function of specific $\mathrm{K}^{+}$channels in sustained high-frequency firing of fast-spiking neocortical interneurons. J. Neurophysiol. 82, 2476-2489.

Ermentrout, B., 1998. Neural networks as spatio-temporal patternforming systems. Rep. Prog. Phys. 61, 353-430.

Fisahn, A., McBain, C.J., 2001. Interneurons unbound. Nat. Rev. Neurosci. 2, 11-23.

Galarreta, M., Hestrin, S., 1999. A network of fast-spiking cells in the cortex connected by electrical synapses. Nature 402, 72-75.

Galarreta, M., Hestrin, S., 2001a. Electrical synapses between GABA-releasing interneurons. Nat. Rev. Neurosci. 2, 425-433.

Galarreta, M., Hestrin, S., 2001b. Spike transmission and synchrony detection in networks of GABAergic interneurons. Science 292, 2295-2299.

Galarreta, M., Hestrin, S., 2002. Electrical and chemical synapses among parvalbumin fast-spiking GABAergic interneurons in adult mouse neocortex. Proc. Natl. Acad. Sci. U.S.A. 99, 12438-12443.

Gibson, J.R., Beierlein, M., Connors, B.W., 1999. Two networks of electrically coupled inhibitory neurons in neocortex. Nature 402, 75-79.

Gray, C.M., Koning, P., Engel, A.K., Singer, W., 1989. Oscillatory responses in cat visual cortex exhibit inter-columnar synchronisation which reflects global stimulus properties. Nature 338, 334-337.

Hormuzdi, S.G., Pais, I., LeBeau, F.E.N., Towers, S.T., Rozov, A., Buhl, E.H., Whittington, M.A., Monyer, H., 2001. Impaired electrical signalling disrupts gamma frequency oscillations in connexin 36-deficient mice. Neuron 31, 487-495.

Lewis, T., Rinzel, J., 2003. Dynamics of spiking neurons connected by both inhibitory and electrical coupling. J. Comp. Neurosci. 14, 283-309.

Pfeuty, B., Mato, G., Golomb, D., Hansel, D., 2003. Electrical synapses and synchrony: the role of intrinsic currents. J. Neurosci. 23, 6280-6294.

Rinzel, J., Ermentrout, B., 1989. Analysis of neural excitability and oscillations. In: Koch, C., Segev, I. (Eds.), Methods in Neuronal Modeling. The MIT Press, Cambridge.

Traub, R.D., Kopell, N., Bibbig, A., Buhl, E.H., LeBeau, F.E.N., Whittington, M.A., 2001. Gap junctions between interneuron dentrites can enhance synchrony of gamma oscillations in distributed networks. J. Neurosci. 21, 9478-9486.

Van Vreeswijk, C.A., Abbott, L.F., Ermentrout, G.B., 1995. Inhibition, not excitation, synchronizes coupled neurons. J. Comp. Neurosci. 1, 303-313.

Wang, X.J., Buzsaki, G., 1996. Gamma oscillation by synaptic inhibition in a hippocampal interneuronal network model. J. Neurosci. $16,6402-6413$. 
Wang, X.J., Rinzel, J., 1992. Alternating and synchronous rhythms in reciprocally inhibitory model neurons. Neural Comput. 4, 8497.

White, J.A., Chow, C.C., Rit, J., Soto-Trevino, C., Kopell, N., 1998. Synchronization and oscillatory dynamics in heteroge- neous, mutually inhibited neurons. J. Comp. Neurosci. 5, 516.

Whittington, M.A., Traub, R.D., Jefferys, J.G.R., 1995. Synchronised oscillations in interneuron networks driven by metabotropic glutamate receptor activation. Nature 373, 612-615. 